\title{
The Total Free Radical Trapping Ability of Cord Blood Plasma in Preterm and Term Babies
}

\author{
J. H. N. LINDEMAN, D. VAN ZOEREN-GROBBEN, J. SCHRIJVER, A. J. SPEEK, \\ B. J. H. M. POORTHUIS, AND H. M. BERGER \\ Department of Pediatrics, University of Leiden, Leiden [J.H.N.L., D.V.Z-G., B.J.H.M.P., H.M.B.] and TNO- \\ CIVO, Toxicology and Nutrition Institute, Zeist [J.S., A.J.S.], The Netherlands
}

\begin{abstract}
The interaction between various antioxidants may be important in protecting the newborn baby against oxygen toxicity. We studied the total radical trapping capacity of the antioxidants in plasma (TRAP) and compared the TRAP level in the preterm and term baby (cord blood) with that in adults. In addition, the concentrations of various known antioxidants were measured and the theoretical contribution of these antioxidants to the TRAP calculated. The measured and calculated TRAP were higher in the newborn babies than the adults. The uric acid concentration was similar in the three groups but the vitamin $C$ concentration was higher and the vitamin $E$ and sulfhydryl concentrations were lower in the newborn babies. In contrast to the adult group, the measured TRAP in the newborn babies did not correlate with the calculated TRAP. This may be due to differences in inhibition or recycling of antioxidants in the newborn and adult groups. Theoretical considerations showed that there may be a large unidentified group of antioxidants that contribute to measured TRAP in plasma. Bilirubin and $\beta$-carotene were measured (higher and lower concentrations, respectively, in the newborn) in an attempt to identify these antioxidants. The efficient plasma radical trapping capacity in the cord blood may partly compensate for deficiencies in other components of the antioxidant defenses, e.g. cellular enzymes, at the time of birth. (Pediatr Res 26: 20-24, 1989)
\end{abstract}

\section{Abbreviations}

TRAP, total radical trapping antioxidant parameter TRAPmeas, measured TRAP

TRAPcalc, calculated TRAP

UNID, unidentified antioxidant

Bronchopulmonary dysplasia, intracerebral hemorrhage, and retinopathy of prematurity are common problems in very low birth wt babies and are major contributors to their high morbidity (1). Despite evidence that these diseases are produced by oxygen toxicity $(2,3)$, attempts to limit their incidence and severity, by prophylactic therapy with the fat-soluble antioxidant vitamin $\mathrm{E}$, have produced conflicting results (4).

The total antioxidant capacity in man is dependent on the synergistic action of various antioxidants, e.g. enzymes such as

Received October 26, 1988; accepted February 27, 1989.

Correspondence H. M. Berger, M.D., University Hospital Leiden, Department of Pediatrics, Neonatal Unit, P.O. Box 9600, 2300 RC Leiden, The Netherlands.

Supported by Milupa Nederland and the Stichting Het Irene Kinderziekenhuis Arnhem grant to H.N. glutathione peroxidase and agents such as vitamin $\mathrm{E}$ and $\mathrm{C}$ and uric acid $(5,6)$. This potentially important interaction between the various antioxidants has not been explored in clinical studies $(6,7)$. A test has been developed to measure the total radical trapping capacity in plasma (7). This TRAP was measured in healthy adults and the contributions of vitamins $E$ and $C$, uric acid and sulfhydryl groups to the TRAP were estimated (7). Subsequently Thurnham et al. studied ill patients and found abnormal radical trapping capacities in adults with rheumatoid arthritis (8) and children with malaria (9).

In the newborn the protective role of these antioxidants and their possible interactions should also be considered (3). We therefore measured the concentration of various plasma antioxidants and the TRAP of newborn babies of varying gestational age.

\section{MATERIALS AND METHODS}

Patients. Venous blood samples were obtained from 17 adults after a 4-h morning fast. All adults were healthy, nonsmoking, Caucasian volunteers: 11 male, six female; age 29 y [mean (SD)] (4). In 38 Caucasian babies, venous cord blood samples were obtained from the separated placenta, within 15 min of their birth. Hemolysis was prevented by gentle aspiration using a syringe and a 19-French gauge needle. The mothers were healthy, and their babies weighed between the 10th and 90th percentile (10) and showed no evidence of birth asphyxia (Table 1). In seven mothers $\beta$-sympathicomimetics $(n=7)$, and indomethacin $(n=2)$ were used to inhibit labor (preliminary analysis showed that these agents did not influence the measurements).

Meausurements. The heparinized blood was immediately centrifuged $(750 \times \mathrm{g}, 10 \mathrm{~min})$ and the plasma stored under argon gas at $-70^{\circ} \mathrm{C}$ until analyzed within $4 \mathrm{wk}$. Preliminary studies confirmed that the TRAP value and the vitamins $C$ and $E$ and sulfhydryl levels $(7,9)$ did not change over this period.

Plasma antioxidants. Uric acid and total bilirubin were measured on a SMAC II automatic analyzer (Technicon Instruments, Tarrytown, NY) by the manufacturer's methods $(11,12)$. Vitamin $\mathrm{E}, \beta$-carotene, and total vitamin $\mathrm{C}$ concentrations were measured by HPLC (13-15). The total plasma sulfhydryl concentration was measured by Ellman's method (16) as adapted by Koster et al. (17) [within $(n=10)$ and between assay $(n=5)$ coefficients of variation were 3 and $1.7 \%$ respectively].

Measurement of TRAP (7). Measurement details are given in Figure 1. The peroxidation of linoleic acid was induced by a water soluble thermolabile free radical initiator $\left(2,2^{\prime}\right.$-azo-bis $(2-$ amidinopropane)-HCl. Peroxidation was initially inhibited (induction phase) by the plasma antioxidants and then progressed rapidly (propagation phase). The duration of the induction phase (an index of the total radical trapping capacity of the plasma) was quantified using the duration of the induction phase produced by a known quantity of an antioxidant with a known 
stoichiometric factor. This value $(\mu \mathrm{mol} / \mathrm{liter})$ was the TRAPmeas.

In addition to the direct measurement of TRAP this parameter was also calculated stoichiometrically (TRAPcalc) (7), by using the experimentally determined efficiency ( $n$ value) of each mol of antioxidant to trap a peroxyl radical, e.g. $n=2.0$ for vitamin $E$ (8). Thus from the measured concentration of the plasma antioxidants: uric acid [UA], vitamin C [VC], vitamin E [VE], and total sulfhydryl groups [SH]:

TRAPcalc $(\mu \mathrm{mol} /$ liter $)=1.3[\mathrm{UA}]+1.7[\mathrm{VC}]+2.0[\mathrm{VE}]+0.2[\mathrm{SH}]$

Although these antioxidants are considered to be the major plasma antioxidants, other compounds may also contribute to the TRAP. These UNID were calculated as TRAPmeas minus TRAPcalc $(7,8)$. Bilirubin (18) and $\beta$-carotene (7) may contribute to this unidentified component and their plasma concentrations were therefore measured (see above).

Statistics. The differences between the results in the three groups were tested by ANOVA. When significant, pair-wise comparisons were carried out using the independent $t$ test, except for the $\beta$-carotene measurements in which a nonparametric test (Kruskal-Wallis) was used. Correlations were calculated by Pearson's method. Values of $p<0.05$ were regarded as significant.

Table 1. Clinical details of newborn babies [mean (SD)]

\begin{tabular}{lll}
\hline & Preterm & \multicolumn{1}{c}{ Term } \\
\hline$n$ & 18 & 20 \\
Gestational age (wk) & $32(3)$ & $39(1)$ \\
Wt percentile & $54(22)$ & $48(27)$ \\
Female & 11 & 12 \\
Apgar score 1 min & $7(3)$ & $9(1)$ \\
Apgar score 5 min & $9(2)$ & $10(1)$ \\
Cord blood pH & $7.36(0.08)$ & $7.33(0.06)$ \\
\hline
\end{tabular}

RESULTS

Table 2 shows the plasma concentrations of the various antioxidants. The uric acid concentration was similar in the three groups but the vitamin $\mathrm{C}$ concentration was higher and the vitamin $\mathrm{E}$ and sulfhydryl concentrations were lower in the preterm and the term babies. The bilirubin levels were higher and the $\beta$-carotene lower in the newborn babies. The fat soluble antioxidants vitamin $\mathrm{E}$ and $\beta$-carotene had, by far, the lowest concentration of the measured antioxidants. The results in the preterm and term babies were not significantly different. There were no gender-related differences in the antioxidant concentrations.

Figure 2 shows the TRAPmeas, TRAPcalc, and the calculated individual trapping capacity of uric acid, vitamin C, sulfhydryl, vitamin $\mathrm{E}$, and the unidentified antioxidants. The TRAPmeas and TRAPcalc were higher in the preterm babies than in the adults. In the term babies only the TRAPcalc was higher than in the adults. The contribution of uric acid to the TRAP was similar in all the groups, but that of vitamin $\mathrm{C}$ higher, and vitamin $\mathrm{E}$

Table 2. Plasma concentrations ( $\mu \mathrm{mol} /$ liter) of the various plasma antioxidants [mean (SD)]

\begin{tabular}{lccc}
\hline & \multicolumn{4}{c}{ Preterm } & \multicolumn{1}{c}{ Term } & Adult \\
\hline Uric acid & $277(68)$ & $317(66)$ & $285(55)$ \\
Vitamin C & $157(56)^{*}$ & $130(55)^{*}$ & $62(16)$ \\
Sulfhydryl & $415(60)^{*}$ & $422(80) \dagger$ & $496(57)$ \\
Vitamin E & $9.1(4.1)^{*}$ & $9.0(4.8)^{*}$ & $21.1(3.1)$ \\
Bilirubin & $30.2(6.6)^{*}$ & $29.7(8.4)^{*}$ & $13.4(5.3)$ \\
$\beta$-Caro- & $0.027(0.05)^{*}$ & $0.007(0.013)^{*}$ & $0.30(0.21)$ \\
tene & & & \\
\hline
\end{tabular}

Preterm or term group significantly different from adult group, ${ }^{*} p<$ $0.001 ; \uparrow p<0.01$

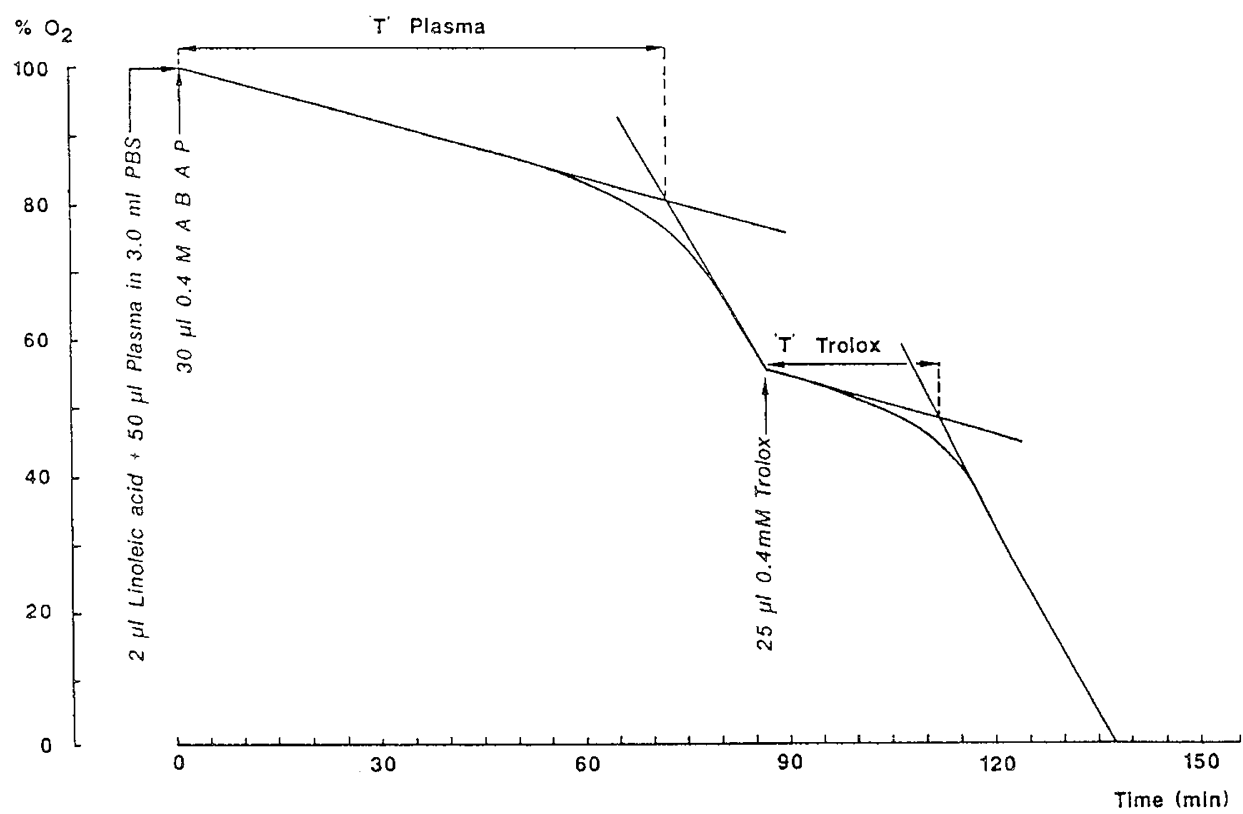

Fig. 1. In the presence of plasma, peroxidation of linoleic acid, induced by $2,2^{\prime}$-azo-bis (2-amidinopropane)-HCI, (ABAP), (Polysciences, Warrington PA) in $10 \mathrm{mM}$ PBS ( $\mathrm{pH} 7.4,37^{\circ} \mathrm{C}$ ) was monitored by oxygen consumption using an oxygen electrode calibrated to read $100 \%$ at ambient oxygen pressure (YSI Biological Oxygen Monitor, model 5300: Yellow Springs Instrument Company, Yellow Springs, OH). The duration of the induction phase (' $T$ ' plasma) was derived as follows: the linear slope of the induction phase and that of the propagation phase (when oxygen consumption is maximal) are extrapolated and their point of intersection is used to measure ' $T$ ' plasma. When approximately $50 \%$ of the oxygen in the reaction medium has been consumed the water soluble vitamin E analogue 6-hydroxy-2,5,7,8-tetramethylchroman-2-carboxylic acid (Trolox, Hoffman-La Roche) was added. This produced a second induction phase ('T' Trolox) which was determined as described for ' $T$ ' plasma. The TRAPmeas was then calculated as follows: TRAPmeas ( $\mu$ mol/liter) $=\mathrm{n}[$ Trolox]('T' plasma/' $\mathrm{T}$ ' Trolox)f; $n=2$ (stoichiometric factor for Trolox); [Trolox] $=0.4 \mathrm{mM}$. ' $\mathrm{T}$ ', induction time measured from recorder trace; $f$, dilution factor plasma/dilution factor Trolox $(=0.5)$. The within $(n=4)$ and between assay $(n=5)$ coefficients of variance of the TRAPmeas were 4.3 and $4.2 \%$ respectively. 


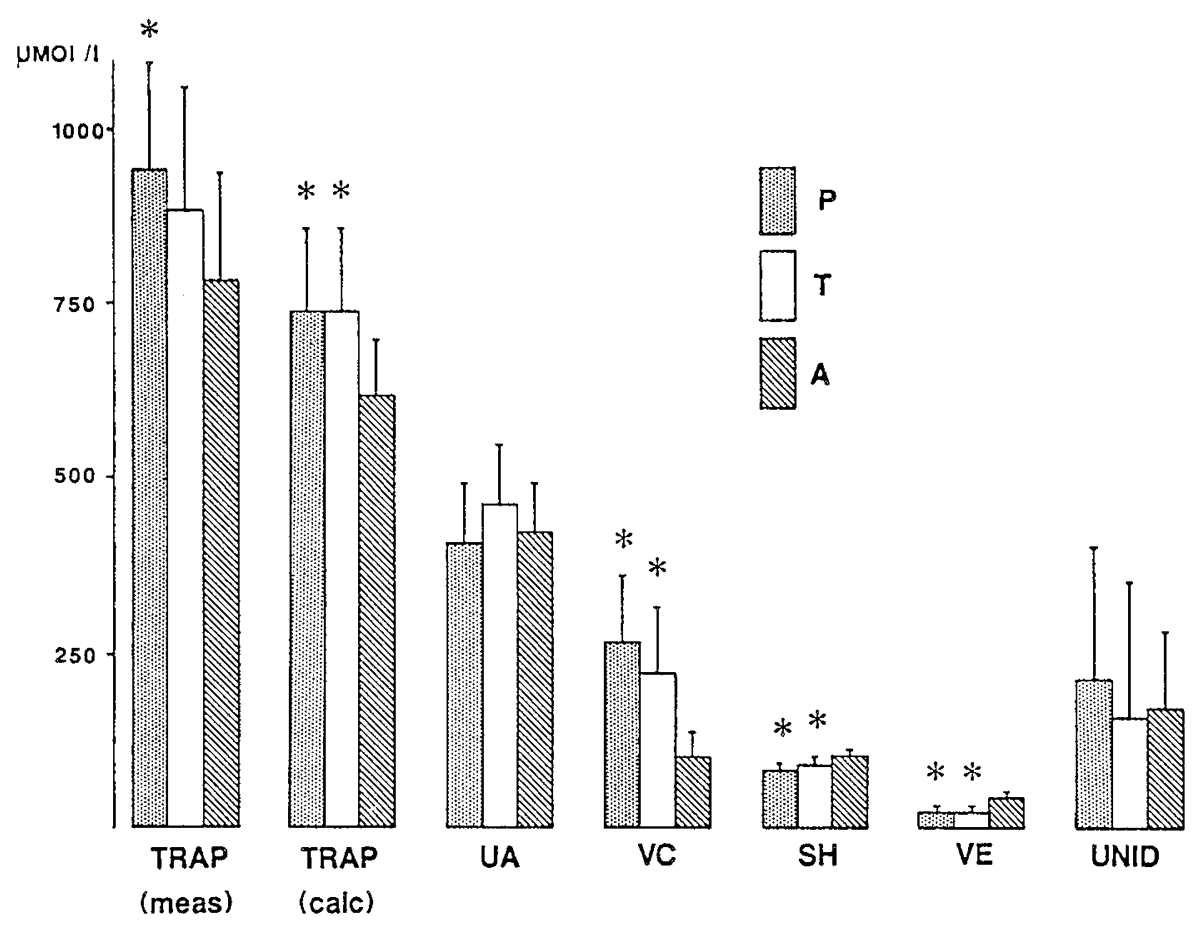

Fig. 2. The TRAPmeas, TRAPcalc, and the calculated individual contributions of uric acid $(U A)$, vitamin $\mathrm{C}(V C)$, total sulfhydryl groups $(S H)$, vitamin $\mathrm{E}(V E)$, and UNID, [mean (SD)] in the preterm $(P)$, term $(T)$, and adult $(A)$ groups. * preterm group or term group significantly different from adult group, $p<0.01$.

and sulfhydryl lower in the newborn babies. The contribution of UNID was similar and large in all groups. The TRAPmeas, TRAPcalc, and antioxidant contributions in the preterm and term babies were not significantly different; and did not correlate with the gestational age (results not shown). Table 3 shows the calculated percent contribution of the antioxidants to the TRAPmeas.

Table 4 shows the correlations of TRAPmeas with TRAPcalc and the calculated trapping capacity of the individual antioxidants. In the adult, unlike the newborn babies, the TRAPmeas correlated with the TRAPcalc and uric acid. The UNID in both the adult and newborn babies correlated with the TRAPmeas. There was a weak negative correlation between TRAPmeas and vitamin $\mathrm{E}$ in the newborn babies $(p<0.04)$.

In an attempt to measure the role of bilirubin in the TRAP, using the stoichiometric factor, $n=2.0$ (19), we calculated its free radical trapping ability [mean (SD)]; preterm; 60 (13), term; 59 (17), and adult; 27 (11) $\mu \mathrm{mol} /$ liter. Recalculating TRAPcalc [TRAPcalc $\mu \mathrm{mol} /$ liter $=1.3[\mathrm{UA}]+1.7[\mathrm{VC}]+2.0[\mathrm{VE}]+0.2[\mathrm{SH}]$ +2.0 [bilirubin]] with these values still leaves a large unidentified component in all the patient groups [mean (SD) preterm: 152 (188), term: 101 (202), adult: 141 (116) $\mu \mathrm{mol} / \mathrm{liter}]$. The plasma concentration of $\beta$-carotene (Table 2) is too low to make a significant contribution to the TRAPcalc.

\section{DISCUSSION}

The pathogenesis of oxygen toxicity in the newborn baby is related to the production of excess amounts of the highly reactive species of oxygen (superoxide- and hydroxyl-free radicals, and hydrogen peroxide) $(2,3)$. This occurs during exposure to high ambient oxygen concentrations, but factors such as iron overload $(3,20)$ and excess xanthine oxidase activity $(21)$ may also be important. The defenses against oxygen toxicity (5) include enzymes, e.g. superoxide dismutase and glutathione peroxidase (22), metal chelators, e.g. transferrin and ceruloplasmin (20), and various antioxidants, e.g. vitamins $\mathrm{E}$ and $\mathrm{C}, \beta$-carotene, sulfhydryl groups, and uric acid (7).
Table 3. Percentage contribution of antioxidants to the TRAPmeas [mean (SD)]

\begin{tabular}{lccc}
\hline & Preterm & Term & Adult \\
\hline Uric acid & $38.3(9.4)$ & $46.7(9.8)$ & $47.4(9.2)$ \\
Vitamin C & $28.5(6.0)$ & $25.1(6.2)$ & $13.4(2.0)$ \\
Sulfhydryl & $8.8(2.1)$ & $9.5(1.7)$ & $12.7(1.5)$ \\
Vitamin E & $1.9(0.4)$ & $2.0(1.1)$ & $5.4(0.8)$ \\
Unidentified & $22.5(19.8)$ & $17.8(21.8)$ & $21.5(14.8)$ \\
\hline
\end{tabular}

Table 4. Correlations of TRAPcalc and calculated trapping capacity of antioxidants with TRAPmeas

\begin{tabular}{lcc}
\hline & \multicolumn{2}{c}{ Correlation coefficient $(r)$} \\
\cline { 2 - 3 } & Newborn $(n=38)$ & Adult $(n=17)$ \\
\hline TRAPcalc & 0.2006 & $0.7414^{*}$ \\
Uric acid & 0.2215 & $0.7320^{*}$ \\
Vitamin C & 0.0752 & -0.0576 \\
Sulfhydryl & 0.1149 & 0.3790 \\
Vitamin E & $-0.3529 \dagger$ & 0.3552 \\
Unidentified & $0.8131^{*}$ & $0.8844^{*}$ \\
\hline
\end{tabular}

${ }^{*} p<0.001$.

In studying the role of the antioxidant defenses in the prevention of oxygen toxicity in the newborn baby, much attention has been paid to the role of vitamin $\mathrm{E}$ and the antioxidant enzymes $(4,22)$ but little attention has been given to the other antioxidants (3). Synergistic interactions occur between the various antioxidants and complete understanding of the total antioxidant defense system in the baby will require measurement of the combined effect of the antioxidants and not just the measurement of their individual cellular or plasma concentrations $(6,7)$. Antioxidants that reduce the rate of production of new radicals, e.g. superoxide dismutase, transferrin, are referred to as primary or preventive antioxidants. Antioxidants that trap radicals and thereby reduce the chain length of oxidation are referred to as 
secondary or chain-breaking antioxidants, e.g. vitamin $\mathrm{E}$ and $\mathrm{C}$ (23). The TRAP provides a means of assessing the total capacity of the chain-breaking antioxidants to prevent lipid peroxidation in plasma.

We found that at birth, well newborn babies have TRAPmeas values equal to and in preterm babies even higher than the adults. The results in the adults were similar to those found in two previous studies $(7,8)$. This efficient plasma-free radical trapping capacity at birth may partly compensate for deficiencies in other antioxidants such as transferrin (20) and superoxide dismutase (22).

The values of the TRAPcalc were considerably lower than the TRAPmeas in all the groups. Two possible explanations for this finding are that either all the plasma antioxidants have not been identified or that the stoichiometric values $(n)$ used to calculate their free radical trapping capacity are too low. Very recently bilirubin has been recognized as being an important antioxidant $(18,19)$. It has a high plasma concentration and a powerful radical trapping capacity $(n=2)$, but including its contribution in the TRAPcalc still left a large unidentified component. The concentration of the fat-soluble $\beta$-carotene in the plasma was too low to explain the remaining gap. However, the plasma proteins are present in high concentrations and only their sulfhydryl groups are used in the formula estimating the TRAPcalc: the amino acids tryptophan, tyrosine, and histidine can also act as antioxidants and may partly account for the unidentified antioxidants (7). Because of the apparently high concentration of unidentified antioxidant it will be important to specifically identify its components and determine what perinatal factors influence their concentrations. The other explanation for the lower TRAPcalc is that the stoichiometric values may be too low. Different workers use different stoichiometric values for the antioxidants, e.g. $n=0.33$ and 2.0 instead of $n=0.2$ and 1.3 for the sulfhydryl groups and uric acid, respectively $(7,24)$ and, furthermore, Wayner et al. (25) have shown that the value for vitamin $\mathrm{C}$ is concentration dependent. However, even if all the contributing antioxidants are identified and their correct stoichiometric values used, the TRAPcalc does not take into account the very important interactions that occur between antioxidants when they work in unison $(6,7)$. For example, the recycling of vitamin $\mathrm{E}$ (with its stronger free radical trapping capacity, $n=$ 2) by vitamin $C(n=1.7)$ is believed to be an important mechanism in defending against lipid peroxidation $(7,26)$. These interactions do occur when the TRAPmeas assay is performed and may explain the greater than predicted free radical trapping capacity in plasma. Therefore, it is possible that the unidentified antioxidant is an artefact. The positive correlation of the unidentified antioxidant with the TRAPmeas is, however, evidence against this point of view.

The correlations between the TRAPmeas and the TRAPcalc and individual antioxidants were different in the adults and the babies. In the studies of Thurnham et al. $(8,9)$ the correlation also varied markedly in different patient groups. They suggested that the inhibition of antioxidant activity, e.g. cation binding of urate (27) or increased prooxidant activity may explain these findings (8). Prooxidant activity due to iron (3) may be particularly important in the newborn plasma as transferrin is highly saturated with iron (28). The unidentified antioxidants showed the strongest correlation with the TRAPmeas in both the newborn and adults emphasizing the need to identify its components.

Despite the possible errors in calculating the exact contribution of the antioxidants to the TRAPmeas they are present in large concentrations and their role in the total antioxidant capacity of the newborn baby requires study. Some of these antioxidants, e.g. uric acid $(29,30)$ have received little attention in the newborn, and others have markedly different plasma concentrations compared to the values in adults.

The uric acid levels in the cord blood were similar to the adult values. Uric acid levels at birth are closely related to the maternal values (31). Uric acid was the major contributor $(38-47 \%)$ to the
TRAPcalc in the newborn and the adult, but only in the adult did it correlate with the TRAPmeas; a possible reason has been mentioned above. Ames et al. (32) hypothesized that uric acid, because of its powerful antioxidant capacity and high plasma concentration, is a major antioxidant in man and protects against aging. We subsequently suggested that uric acid also plays an important role at birth when the baby emerges from the relatively hypoxic conditions in utero into the raised oxygen levels of our environment (29). The high uric acid levels at this time contrast with the low levels of other components (e.g. transferrin, superoxide dismutase) of the antioxidant defenses $(20,22)$.

The vitamin $C$ levels in the babies were two to three times higher than in the adults. Vitamin $\mathrm{C}$ is actively transported across the placenta (33) and the maternal/fetal ratio is 0.5 . Vitamin C was calculated as contributing only $13 \%$ in the adults but $25-$ $29 \%$ of the TRAP in the babies, and was thus the second largest contributor in the newborn. Vitamin $\mathrm{C}$, despite its large calculated contribution, however, had the weakest correlation of all the antioxidants with TRAPmeas. We, like Thurnham et al. (8), measured the total vitamin $C$ rather than just the reduced ascorbate as done by Wayner et al. (7). Only $5 \%$ of vitamin $C$ is in the oxidized form in normal adult plasma (34): if our assumption that this is so in the newborn baby is not valid, the calculated contribution and correlation findings are incorrect.

The sulfhydryl groups have the highest plasma concentration of all antioxidants, but they are relatively ineffective antioxidants and their percent contribution to the TRAP is low. Wayner et al. (7) showed in vitro that the sulfhydryl groups are the "most expendable" of the secondary antioxidants and are the first to be consumed in the TRAP assay. The higher plasma protein levels (the major source of sulfhydryl groups in the plasma) in the adult explains their higher sulfhydryl group concentrations. Interestingly, although plasma protein levels are related to gestational age the sulfhydryl concentrations were similar in the preterm and term babies.

Vitamin $E$ is the lowest contributor $(2-5 \%)$ to the TRAP. The concentration of this fat soluble vitamin is low in cord blood, which has low fat levels, and its contribution to the TRAP is lower in the newborn babies. However, vitamin $\mathrm{E}$ is the major chain-breaking antioxidant in the lipid membrane and thus an essential member of the antioxidant defenses. The recycling of the limited vitamin $E$ concentrations by vitamin $C$, which is able to reduce the tocopherol radical in the lipid phase, is believed to be very important $(7,26)$. The high cord blood vitamin $C$ levels in the newborn may result in maximal use of the limited vitamin E concentrations. Uric acid and sulfhydryl groups, unlike vitamin C, are unable to regenerate vitamin E and these "sacrificial" antioxidants act more by sparing vitamin $\mathrm{E}$ than by recycling (7).

Free radical chain oxidation and the interaction of various antioxidants are now attracting the attention of nutritionists (35). The important role of vitamin $\mathrm{C}$ and uric acid in the TRAP at birth suggests that changes in their concentration due to nutritional and iatrogenic factors may influence the susceptibility of the baby to oxygen toxicity. We have shown that the vitamin $\mathrm{C}$ content of human milk falls when it is processed (36) and reported a marked postnatal fall in plasma uric acid levels in the preterm baby (30). The TRAP test uses only $50 \mu$ l of plasma and may prove to be a useful test for assessing the postnatal changes in the total radical trapping capacity of the plasma in the preterm baby.

Acknowledgments. The authors thank Drs. D. Thurnham and S. Koottahep for advice on the method; E. Lentjes (Department of Clinical Chemistry), R. Moison, and E. Houdkamp for their ever increasing help with the free radical project; Prof van Houwelingen, Department of Medical Statistics for help in the design of the study and the analysis of the results; and Laura van Berlo and her midwifes for always assisting us. The figures were drawn by our audio-visual unit and the manuscript prepared by 
Mirjam Silverentand and Ineke Haasnoot. Trolox was kindly supplied by Hoffman-La Roche, Mijdrecht, The Netherlands.

\section{REFERENCES}

1. Usher R 1987 Extreme prematurity. In: Avery GA (ed) Neonatology. JB Lippincott Company, Philadelphia, pp 264-298

2. Phelps DL 1982 Neonatal oxygen toxicity-is it preventable? Pediatr Clin North Am 29:1233-1240

3. Wispe JR, Roberts RJ 1987 Molecular basis of pulmonary oxygen toxicity. Clin Perinatol 14:651-666

4. Karp WB, Robertson AF 1986 Vitamin E in neonatology. Adv Pediatr 33:127148

5. Freeman BA, Crapo JD 1982 Free radicals and tissue injury. Lab Invest 47:412-426

6. Willson RL 1983 Free radical protection: why vitamin $E$, not vitamin $C, \beta$ carotene or glutathione? In: Biology of Vitamin E. Ciba Foundation symposium 101. Pitman Books, London, pp 19-44

7. Wayner DDM, Burton GW, Ingold KU, Barclay LRC, Locke S 1987 The relative contributions of vitamin $\mathrm{E}$, urate, ascorbate and proteins to the total radical trapping antioxidant activity of human blood plasma. Biochim Biophys Acta 924:408-419

8. Thurnham DI, Situnayake RD, Koottathep S, McConkey B, Davies M 1987 Antioxidant status measured by the TRAP assay in rheumatoid arthritis. In: Rice Evans C (ed) Free Radicals, Oxidant Stress and Drug Action. Richelieu Press, London, pp 169-192

9. Thurnham DI, Koottathep S, Adelekan DA 1988 Chain-breaking antioxidants in the blood of malaria infected children. In: Rice Evans C, Dormandy $T$ (eds) Free Radicals: Chemistry, Pathology and Medicine. Richelieu Press, London, pp 161-185

10. Kloosterman GJ 1970 On intrauterine growth. Int J Gynaecol Obstet 8:895912

11. Musser WA, Ostigasa C 1966 Automated determination of uric acid by the hydroxylamine method. Tech Bull Reg Med Tech 30:21-25

12. Gambino RS, Schreiber H 1964 The measurement and fractionation of bilirubin on the auto analyzer by the method of Jendrassik and Grof. In: Automation in Analytical Chemistry, Technicon Symposia. Mediac Inc, White Plains, NY

13. Tangney CC, McNair HN, Driskell JA 1981 Quantitation of individual tocopherols in plasma, platelets, lipids and livers by high-performance liquid chromatography. J Chromatogr 224:389-397

14. Speek AJ, Temalilwa CA, Schrijver J 1986 Determination of $\beta$-carotene content and vitamin A activity of vegetables by high performance liquid chromatography and spectrophotometry. Food Chem 19:65-74

15. Speek AJ, Schrijver J, Schreurs WHP 1984 Fluormetric determination of total vitamin $C$ in whole blood by high performance liquid chromatography with pre-column derivatization. J Chromatogr 305:53-60

16. Ellman GL 1959 Tissue sulfhydryl groups. Arch Biochem Biophys 82:70-77

17. Koster JP, Biemond P, Swaak AJG 1986 Intracellular and extracellular sulfhy- dryl levels in rheumatoid arthritis. Ann Rheum Dis 45:44-46

18. Stocker R, Yamamoto Y, McDonagh AF, Glazer AN, Ames BN 1987 Bilirubin is an antioxidant of possible physiological importance. Science 235:10431046

19. Stocker R, Glazer AN, Ames BN 1987 Antioxidant activity of albumin-bound bilirubin. Proc Natl Acad 84:5918-5922

20. Sullivan JL, Newton RB 1988 Serum antioxidant activity in neonates. Arch Dis Child 63:748-750

21. Saugstad 1988 Hypoxanthine as an indicator of hypoxia: its role in health and disease through free radical production. Pediatr Res 23:143-150

22. Frank L, Sosenko IRS 1987 Development of lung antioxidant enzyme system in late gestation: possible implications for the prematurely born infant. J Pediatr 110:9-14

23. Slater TF, Cheeseman KH, Davies MJ, Proudfoot K, Xin W 1987 Free radical mechanisms in relation to tissue injury. Proc Nutr Soc 46:1-12

24. Niki E, Saito M, Yoshikawa Y, Yamamoto Y, Kamiya Y 1986 Oxidation of lipids. 12. Inhibition of oxidation of soybean phosphatidylcholine and methyl linoleate in aqueous dispersions by uric acid. Bull Chem Soc Jpn 59:471477

25. Wayner DDM, Burton GW, Ingold KU 1986 The antioxidant efficiency of vitamin $C$ is concentration-dependent. Biochim Biophys Acta 884:119-123

26. Packer JE, Slater TF, Willson RL 1979 Direct observation of a free radical interaction between vitamin $\mathrm{E}$ and vitamin $\mathrm{C}$. Nature 278:737-738

27. Lam KW, Fong D, Lee A, Ming K, Liu 1984 Inhibition of ascorbate oxidation by urate. J Inorg Biochem 22:241-248

28. Scott PH, Berger HM, Kenward C, Scott P, Wharton BA 1975 Effect of gestational age and intrauterine nutrition on the plasma transferrin and iron in the newborn. Arch Dis Child 80:796-798

29. Berger HM, Van Zoeren D, Karsdon J 1985 The antioxidant capacity of uric acid and adaptation to a terrestrial environment. Spec Sci Technol 9:83-84

30. Berger HM, Brand EC, Van Zoeren D, Boot W, Stijnen T 1984 Antioxidants in the Newborn: What About Uric Acid? In: Clinch J, Matthews T (eds) Tenth European Congress of Perinatal Medicine. ETA Publications Ltd, Dublin, p 39(abstr)

31. Wharton BA, Bassi U, Gough G, Williams A 1971 Clinical value of plasma creatine kinase and uric acid levels during the first week of life. Arch Dis Child 46:356-362

32. Ames BN, Cathart R, Schwiers E, Hochstein P 1981 Uric acid provides an antioxidant defence in humans against oxidant- and radical-caused aging and cancer: a hypothesis. Proc Natl Sci USA 78:6858-6862

33. Schanler RJ, Nichols BL 1985 The water-soluble vitamins C, B1, B2, B6 and niacin. In: Tsang RC (ed) Vitamin and Mineral Requirements in Preterm Infants. Marcel Dekker Inc, New York, pp 39-62

34. Lee W, Davis KA, Rettmer RL, Labbe RF 1988 Ascorbic acid status: biochemical and clinical considerations. Am J Clin Nutr 48:286-290

35. Editorial 1988 Inhibition of free radical chain by $\alpha$ tocopherol and other plasma antioxidants. Nutr Rev 46:206-207

36. Van Zoeren D, Van den Berg H, Schrijvers J, Berger HM 1987 Effect of storage, pasteurisation and tube feeding on the vitamins in expressed human milk. Arch Dis Child 62:161-165 